\title{
Programa de screening auditivo neonatal universal. Experiencia de más de 10 años
}

\section{Universal newborn hearing screening program. Experience over 10 years}

\author{
Karin Krauss $\mathbf{M}^{1}$, Claudia Heider $\mathbf{C}^{1}$, Gonzalo Nazar $\mathbf{M}^{2}$, Gloria Ribalta L², Mónica Sierra G³.
}

\section{RESUMEN}

Introducción: La implementación de Programas de Evaluación Auditiva Neonatal (PEAN) eficientes permite mejorar la pesquisa de recién nacidos (RN) con hipoacusia congénita $(H C)$, facilitando una intervención temprana.

Objetivo: Describir los resultados del PEAN universal Ilevado a cabo por el Departamento de Otorrinolaringología de Clínica Las Condes.

Material y método: Estudio descriptivo, retrospectivo. Se estudiaron los resultados del programa y las variables: género, edad de diagnóstico, edad gestacional al parto y factores de riesgo asociados al desarrollo de HC en RN evaluados entre mayo 2001 y junio 2012.

Resultados: Fueron evaluados 18.224 RN. Reprobaron esta evaluación 809 RN (4,43\%). Se diagnostican 70 HC (56\% varones, $44 \%$ mujeres), con una incidencia general estimada de 3-4 cada 1.000 RN en este establecimiento. 54,3\% correspondió a HC unilaterales y $45,7 \%$ a bilaterales, siendo éstas en $90 \%$ leves a moderadas. El factor de riesgo mayormente asociado fue presencia de anomalías craneofaciales en $38,5 \%$ seguido de un peso nacimiento $<1.500$ gr. en $10 \%$.

Discusión y conclusiones: Es imperativo la realización de PEAN universales para diagnosticar a la población general susceptible a desarrollar HC y más aún en aquellas poblaciones donde se concentran los factores de riesgo.

Palabras clave: Screening auditivo neonatal, hipoacusia congénita, tamizaje auditivo.

\begin{abstract}
Introduction: Efficient universal newborn hearing screening program (UNHSP) Implementation allows the inquest improvement of newborns with congenital hearing loss (CHL), facilitating an early intervention.

Aim: To describe the results of the UNHSP carried out by the Department of Otorhinolaryngology of Clinica Las Condes.
\end{abstract}

\footnotetext{
${ }^{1}$ Médicos en Programa de Capacitación en Otorrinolaringología, Clínica Las Condes.

${ }^{2}$ Médico. Departamento de Otorrinolaringología, Clínica Las Condes.

${ }^{3}$ Tecnólogo Médico. Departamento de Otorrinolaringología, Clínica Las Condes.
} 
Material and method: Descriptive, retrospective study. We studied the program results and variables: sex, diagnosis age, birth age in weeks and risk factors associated with development CHL in newborns evaluated between May, 2001 and June, 2012.

Results: 18.224 newborns were evaluated. 809 reproved this evaluation (4.43\%). We diagnosed $70 \mathrm{CHL}$ (56\% males, 44\% women), with an incidence estimated of 3-4 cases every 1.000 newborns. 54.3\% corresponded to unilateral CHL and $45.7 \%$ bilateral, $90 \%$ of this $\mathrm{CHL}$ were slight to moderate. The presence of craniofacial anomalies was the main associate risk factor in $38,5 \%$ followed by a weight birth $<1.500 \mathrm{gr}$. in a $10 \%$.

Discussion and conclusions: Is imperative the accomplishment of UNHSP to diagnose the population at risk of developing CHL and even more in those populations where the risk factors are concentrated.

Key word: Newborn Hearing Screening, Congenital Hearing Loss, Hearing Screening.

\section{INTRODUCCIÓN}

La hipoacusia congénita es una patología con una importante incidencia en la población neonatal, siendo en Chile incluso más frecuente que la fenilquetonuria clásica y el hipotiroidismo congénito, patologías para las que se realiza pesquisa neonatal universal ${ }^{1}$.

En nuestro país aún no existen políticas a nivel nacional para realizar detección universal de hipoacusia congénita. Países vecinos, con desarrollo similar al nuestro, incluso con ingreso per cápita menor, tienen desde hace años leyes que hacen obligatoria la detección de hipoacusia en todo recién nacido, lo que les ha permitido rehabilitar a sus niños hipoacúsicos e insertarlos en la sociedad como normooyentes en forma eficaz.

A pesar de no contar con legislación en el tema, existen instituciones que por diferentes motivos han decidido implementar programas de detección universal en forma autónoma, éste es el caso de nuestro centro y de otros, tanto privados como públicos, ubicados en distintas regiones de nuestro país.

Se ha estimado que aproximadamente la mitad de los RN con HC no tienen factores de riesgo identificables por lo cual la realización de programas de pesquisa universal son la mejor estrategia para poder diagnosticar esta patología y realizar las intervenciones precozmente? ${ }^{2}$.

En Clínica Las Condes (CLC), el programa de evaluación auditiva neonatal se inició en el año 2001 y hasta la fecha ha evaluado a más de 18.000 niños, alcanzando desde el año 2007 una cobertura del $100 \%$ de los recién nacidos vivos en la clínica. Nuestro desafío actual se enfoca en lograr que aquellos recién nacidos cuya evaluación inicial se encuentre alterada reciban precozmente una segunda evaluación, diagnóstico y derivación oportuna permitiendo una adecuada intervención que facilite un desarrollo lingüístico normal.

\section{OBJETIVO}

Describir los resultados del PEAN universal llevado a cabo por el Departamento de Otorrinolaringología de Clínica Las Condes.

\section{MATERIAL Y MÉTODO}

Estudio descriptivo, retrospectivo. Se estudiaron los resultados del programa y las variables: género, edad de diagnóstico, edad gestacional al parto y factores de riesgo asociados al desarrollo de hipoacusia congénita en RN evaluados entre mayo 2001 y junio 2012, nacidos en la ciudad de Santiago, en los Departamentos de Pediatría y Unidad de Tratamientos Intermedios Neonatales de Clínica Las Condes y cuyo registro en la base de datos del PEAN se encontrará completo. Existe un protocolo interno creado para la evaluación de los RN dependiendo de su procedencia que se describe a continuación:

\section{Protocolo Screening Auditivo Neonatal Universal en CLC}

- Recién Nacidos sin factores de riesgo de hipoacusia: Sala Cuna: (Figura 1) 
Fueron evaluados con Emisiones Oto-Acústicas (EOA) por Productos de Distorsión (PD) mediante el equipo GSI AUDIOscreener ${ }^{\circledR}$, después de las 36 horas de vida. Las frecuencias estudiadas fueron 2, 3, 4 y $5 \mathrm{Khz}$., cada una estimulada con tonos de $65 \mathrm{~dB}$ SPL (L1) y $55 \mathrm{~dB}$ SPL (L2). Los criterios de aprobación del equipo son una relación señal/ruido mayor 0 igual a $6 \mathrm{~dB}$ y el pasar al menos 3 de las 4 frecuencias.

Cuando el paciente reprueba el tamizaje, es reevaluado nuevamente al mes de vida para repetir las EOA por PD. Lo que fue definido como el primer control posterior al screening. Si el paciente no pasa este control, es derivado a estudio audiológico completo con Potenciales
Evocados Auditivos de Tronco Diagnósticos (PEAT-D), también conocidos como BERA o ABR clínicos, e impedanciometría. Esta evaluación se consideró como segundo control.

Finalmente si este último estudio resulta alterado, diagnosticando una hipoacusia sensorioneural cuando se obtiene un registro de onda $\mathrm{V}$ por sobre los $25 \mathrm{~dB}$, utilizando tonos click (2 a 4 Khz.), el paciente es enviado para evaluación por otorrinolaringólogo, tercer control.

- Recién Nacidos con factores de riesgo de hipoacusia: UTI Neonatología (Figura 2)

Este protocolo se inició en enero de 2007 a cargo del Departamento de Otorrinolaringología.

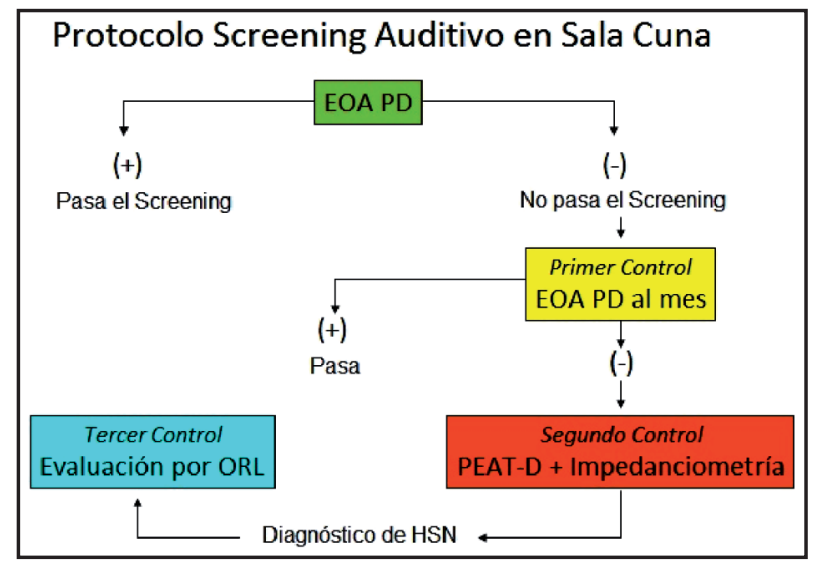

Figura 1. Algoritmo de screening auditivo utilizado en sala cuna CLC. EOA PD: Emisiones otoacústicas por productos de distorsión. PEAT-D: Potencial Evocado Auditivo Diagnóstico. HSN: Hipoacusia sensorioneural. ORL: Otorrinolaringólogo.

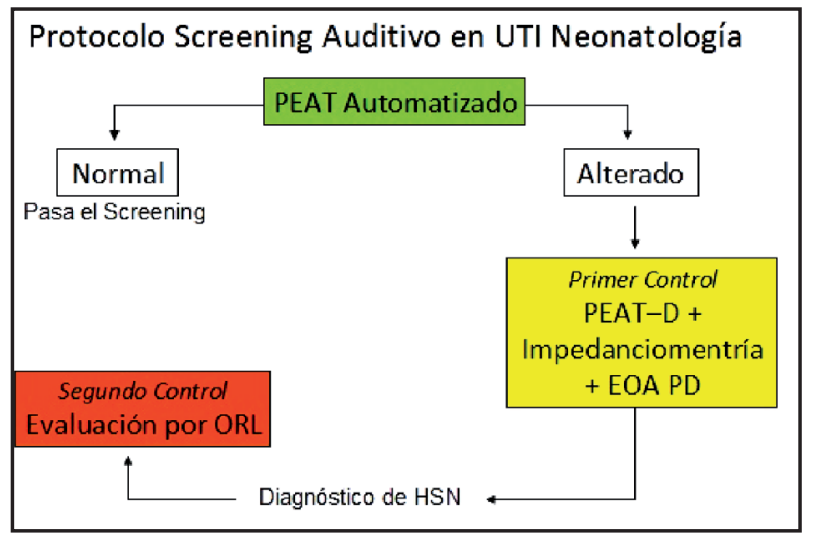

Figura 2. Algoritmo de screening auditivo utilizado en UTI neonatología CLC. PEAT: Potencial Evocado Auditivo. PEAT-D: Potencial Evocado Auditivo Diagnóstico. EOA PD: Emisiones Otoacústicas por productos de distorsión. HSN: Hipoacusia sensorioneural. ORL: Otorrinolaringólogo. 
Previamente se realizaba screening a este grupo de recién nacidos con otro protocolo y a cargo de otro departamento de la clínica (neurología), por lo que la casuística previa no fue considerada.

Fueron evaluados con PEAT automatizados mediante el equipo GSI AUDIOscreener ${ }^{\circledast}$. Para ello se utilizó tono click de rarefacción a $35 \mathrm{~dB}$ HL con criterio Pass de Fsp mayor 0 igual a 3,2 .

Si el paciente reprueba este tamizaje, es derivado directamente a estudio audiológico con PEAT-D, EOA por PD e impedanciometría. Lo que fue considerado como primer control posterior al screening.

Si este último estudio diagnóstico resulta alterado, obteniendo un registro de onda $V$ por sobre los $30 \mathrm{~dB}$, utilizando tonos click (2 a $4 \mathrm{Khz}$.) el paciente es enviado para evaluación por otorrinolaringólogo, completando un segundo control.

\section{RESULTADOS}

Durante el periodo estudiado fueron evaluados en total 18.226 RN, 17.593 provenientes de sala cuna, 633 de UTI. Fueron excluidos del estudio 4 pacientes, dos de cada grupo de evaluación, debido a que no se contaba con el registro completo de sus datos.

Consideramos que se alcanzó la cobertura total a partir del año 2007, ya que no se cuenta con los datos del tamizaje en UTI previo a la implementación del protocolo actual para dicho grupo por el Departamento de Otorrinolaringología.

Reprobaron esta evaluación 814 RN $(4,43 \%)$. 648 correspondientes a SC, 166 de UTI. En las Figuras 3 y 4 se describen los resultados desglosados para cada protocolo.

Se diagnostican $70 \mathrm{HC}$ (56\% varones, 44\% mujeres), con una incidencia estimada de 3-4 casos de HC cada 1.000 RNV en este establecimiento (Figura 5).

$54,3 \%$ correspondió a $\mathrm{HC}$ unilaterales y $45,7 \%$ a bilaterales, siendo éstas en $90 \%$ leves a moderadas (Figura 6).

La mediana para la edad de diagnóstico de HC en el grupo SC correspondió a 42 días con un intervalo entre 5-239 días, en este grupo el $87 \%$ de los

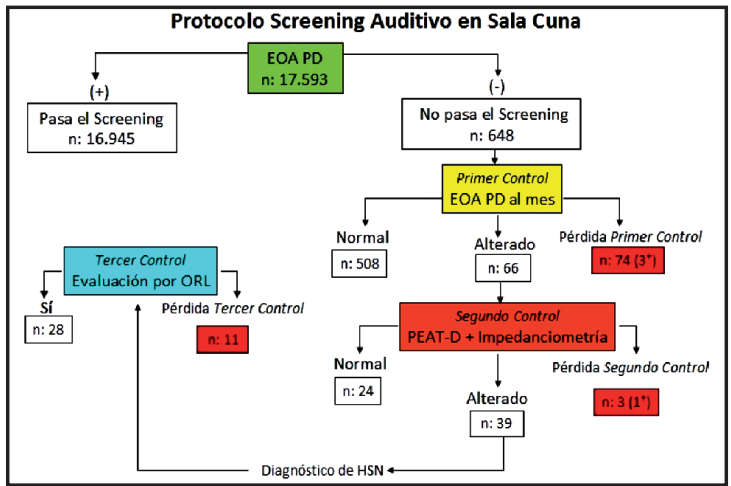

Figura 3. Resultados protocolo screening auditivo en sala cuna CLC. PEAT: EOA PD: Emisiones Otoacústicas por productos de distorsión. PEAT-D: Potencial Evocado Auditivo Diagnóstico. HSN: Hipoacusia sensorioneural. ORL: Otorrinolaringólogo. †Pacientes perdidos por fallecimiento.

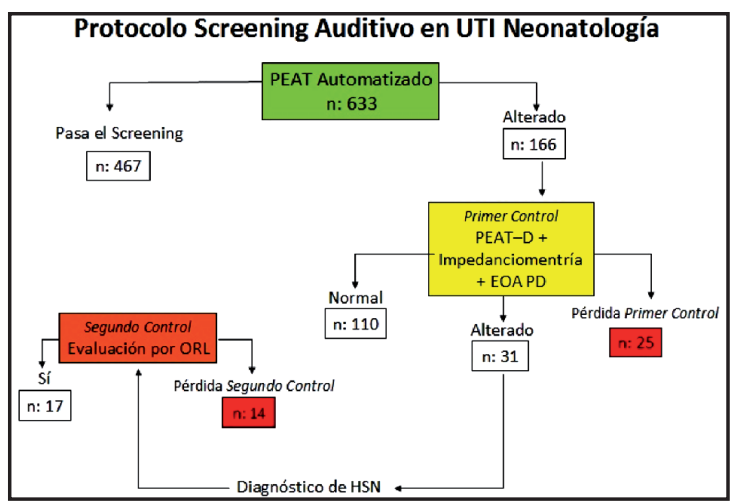

Figura 4. Resultados protocolo screening auditivo en UTI neonatología CLC. PEAT: Potencial Evocado Auditivo. PEAT-D: Potencial Evocado Auditivo Diagnóstico. EOA PD: Emisiones Otoacústicas por productos de distorsión. HSN: Hipoacusia sensorioneural. ORL: Otorrinolaringólogo.

niños fue diagnosticado antes de los 90 días. Para el grupo UTI la mediana fue de 55 días con un intervalo de 4-169 días, en este caso el 83\% de los niños fue diagnosticado antes de los 90 días (Figura 7).

En cuanto a la edad gestacional al momento del parto, la mediana para el grupo SC fue de 38 semanas con un intervalo entre 30-40 semanas y el promedio para el grupo UTI de $34 \pm 3,6$ semanas (25-40 semanas) (Figura 8). Respecto a la presencia de factores de riesgo en la cohorte estudiada, en la Tabla 1 se describen las frecuencias absolutas y relativas. Debido a que se trata de un estudio retrospectivo, se consideraron los factores de riesgo según los criterios del Joint Comitte on Infant 


\begin{tabular}{|c|c|c|c|c|}
\hline & $\begin{array}{l}\text { Total Pacientes } \\
\text { Evaluados }\end{array}$ & $\begin{array}{c}\text { Diagnóstico de } \\
\text { HC }\end{array}$ & Porcentaje & $\begin{array}{c}\text { Tasa de incidencial } \\
1.000 \mathrm{RNV}\end{array}$ \\
\hline Total & 18.226 & 70 & $0,38 \%$ & $3-4$ \\
\hline Unilateral & & 38 & $0,21 \%$ & 2 \\
\hline Bilateral & & 32 & $0,17 \%$ & $1-2$ \\
\hline Sala Cuna & 17.593 & 39 & $0,22 \%$ & 2 \\
\hline Unilateral & & 19 & $0,11 \%$ & 1 \\
\hline Bilateral & & 20 & $0,11 \%$ & 1 \\
\hline UTI & 633 & 31 & $4,80 \%$ & 49 \\
\hline Unilateral & & 19 & $3 \%$ & 30 \\
\hline Bilateral & & 12 & $1,80 \%$ & 19 \\
\hline
\end{tabular}

Figura 5. Tasa incidencia hipoacusia congénita según lugar de diagnóstico. (HC). RNV: Recién nacidos vivos.

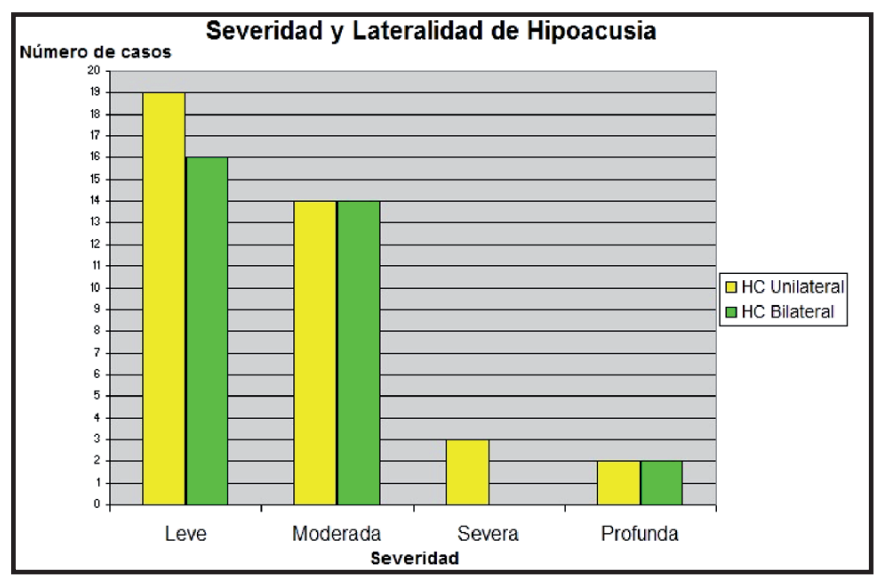

Figura 6. Severidad y lateralidad de hipoacusia (HC). Para determinar la severidad de hipoacusia se consideraron Ios siguientes valores: leve: $\geq 40 \mathrm{~dB}$; Moderada: 41-70 dB; Severa: 71-89 dB; Profunda: $\geq 90 \mathrm{~dB}$.

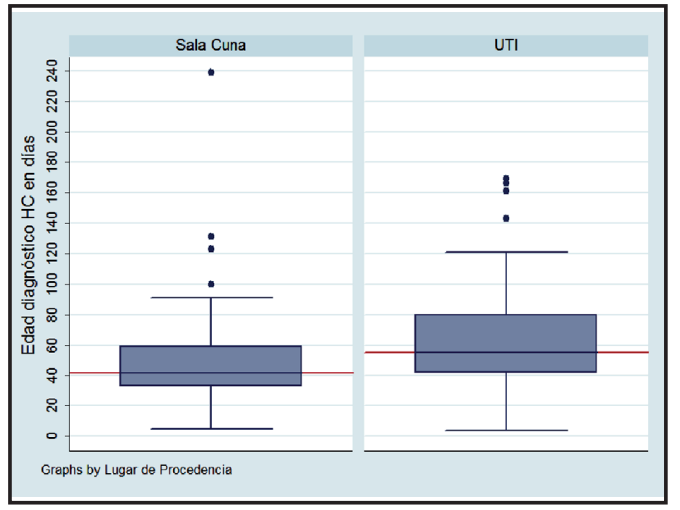

Figura 7. Blox Plot que describe edad del diagnóstico HC en días en relación a la procedencia de los RN.

Hearing $(\mathrm{JCIH})$ de $1990^{5}$, ya que eran los criterios que regían al momento de iniciar el programa en nuestra institución.

Se evidenció una diferencia estadísticamente

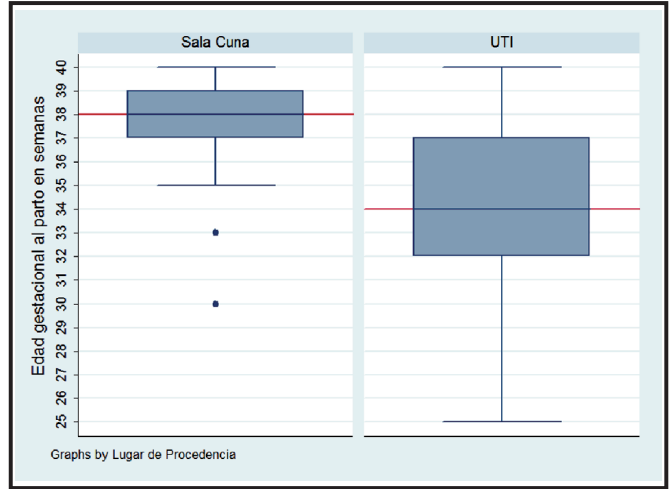

Figura 8. Blox Plot que describe edad gestacional (semanas) al parto en relación a procedencia de los RN.

significativa en cuanto a la procedencia del RN en relación a los factores de riesgo: 1. Anomalías craneofaciales, 2 . Peso $<1.500$ grs. y 3 . Ventilación mecánica (Tabla 2). 
Tabla 1. Prevalencia de factores de riesgo para hipoacusia congénita según los criterios del Joint Comitte on Infant Hearing de 1990

\begin{tabular}{|l|c|c|}
\hline \multicolumn{1}{|c|}{ Factor de riesgo } & F. absoluta & F. relativa \\
\hline & & $38,57 \%$ \\
\hline Anomalías craneofaciales & 27 & $10 \%$ \\
\hline Peso $<1.500$ gr & 7 & $10 \%$ \\
\hline V. mecánica & 7 & $5,71 \%$ \\
\hline Antecedentes familiares & 4 & $5,71 \%$ \\
\hline Apgar & 4 & $4,29 \%$ \\
\hline Hallazgos SD asocian a HC & 3 & $1,43 \%$ \\
\hline TORCH & 1 & $1,43 \%$ \\
\hline Hiperbilirrubinemia & 1 & \\
\hline
\end{tabular}

Anomalías craneofaciales, incluyendo las del pabellón auricular, conducto auditivo externo, fositas preauriculares y anomalías del hueso temporal. Ventilación mecánica durante al menos 5 días. Antecedentes familiares de hipoacusia en familiares de primer grado. Apgar 0 a 4 al minuto 00 a 6 a los 5 minutos. Hiperbilirrubinemia que requirió exanguineotransfusión.

Tabla 2. Factores de riesgo con diferencia estadísticamente significativa según procedencia del RN

\begin{tabular}{|l|c|c|c|}
\hline Factor de riesgo & F. Absoluta SC & F. Absoluta UTI & Valor $p$ \\
\hline Peso $<1.500 \mathrm{gr}$ & 0 & 7 & 0,002 \\
\hline Apgar & 0 & 4 & 0,021 \\
\hline V. Mecánica & 1 & 6 & 0,020 \\
\hline
\end{tabular}

SC: Sala Cuna, UTI: Unidad de tratamiento intermedios neonatales.

Finalmente se estimó que la pérdida de seguimiento correspondió a $13,6 \%$ en el grupo SC y a $23,5 \%$ en el grupo UTI, siendo el porcentaje total de pérdida $15,6 \%$.

\section{DISCUSIÓN}

Al igual que datos publicados tanto a nivel internacional como en revisiones nacionales la tasa de incidencia de hipoacusia en nuestro centro de 3-4 casos cada 1.000 RNV se correlaciona con la tasa de 1-3 casos cada 1.000 RNV4 estimada por el Joint Committe on Infant Hearing. Si considera- mos dichas tasas desglosadas por procedencia, podemos ver que en el grupo UTI ésta se dispara a un nivel altísimo, de 49 casos cada 1.000 RNV. Este incremento tiene relación con que la Unidad de Tratamiento Intermedios Neonatal de Clínica Las Condes corresponde a una unidad de alta complejidad, centro de referencia en la Región Metropolitana, que atiende recién nacidos de alto riesgo cuyo pronóstico y asociación de factores de riesgo los hace más susceptibles al desarrollo de patología auditiva.

En cuanto a la severidad y lateralidad de la hipoacusia, una revisión anterior realizada en Clínica 
Las Condes con una muestra menor, se describe una distribución muy similar a los resultados actuales ${ }^{6}$. Cabe destacar que en nuestro centro la clasificación de la severidad de la hipoacusia ha sido modificada de aquella propuesta por Sociedad Británica de Audiología y Asociación Británica de Profesores de Sordos y que actualmente rige la clasificación en la Guía Clínica Hipoacusia Neurosensorial Bilateral del Prematuro"11, esto debido a que consideramos que dicha clasificación subestima la población de RN con hipoacusias profundas (entre 90 y $95 \mathrm{~dB}$ ) y además porque los equipos para medición de potenciales evocados auditivos de tronco diagnósticos estiman un rango de intensidad sólo hasta $90 \mathrm{~dB}$, por lo cual un valor de 95 $\mathrm{dB}$ queda fuera del rango teórico evaluable.

Todos los RN fueron tamizados antes del mes de vida y en el $85 \%$ diagnosticados antes de los 3 meses. El retraso en el $15 \%$ restante de RN puede explicarse debido a la concomitancia de patologías severas de riesgo vital que requirieron ser corregidas y estabilizadas previa evaluación auditiva.

En nuestra revisión la presencia de anomalías craneofaciales, un peso de nacimiento menor a 1.500 gramos y el uso de ventilación mecánica por al menos 5 días fueron los factores de riesgo más frecuen- temente relacionados, lo que se correlaciona con lo descrito en la literatura ${ }^{4,10}$. Existió una diferencia importante en relación a la presencia de hiperbilirrubinemia, que en nuestra cohorte fue muy escasa. Esto probablemente se deba a que se consideró como criterio que la gravedad de ésta conllevara a la indicación de exanguinotransfusión ${ }^{5}$, criterio que probablemente no ha sido considerado en algunas revisiones en las cuales la sola presencia de hiperbilirrubinemia, independiente de su severidad e indicación de tratamiento, fue adjudicada como factor de riesg $0^{6,8,9}$.

En cuanto a la pérdida en el seguimiento, consideramos que $15,6 \%$ es una cifra aceptable si la comparamos con las cifras internacionales en las cuales se describe una pérdida de hasta $50 \%{ }^{3}$. Este tema será tratado en extenso en otro trabajo de investigación realizado en Clínica Las Condes.

Finalmente debemos reconocer que al tratarse de una revisión retrospectiva estamos conscientes de la probable presencia de sesgo del recuerdo. Además si consideráramos los nuevos criterios para hipoacusia congénita establecidos en el Possition Statement del $\mathrm{JCIH}$ del 2007 (Tabla 3) probablemente nuestros resultados serían distintos, pero dado que la cohorte se inició previa al año 2007, se consideraron los criterios

Tabla 3. Factores de riesgo asociados con hipoacusias infantiles: congénita permanente, de aparición tardía o progresiva

\begin{tabular}{|l|}
\hline Los factores de riesgo que son marcados con un ${ }^{*}$ son de mayor preocupación para hipoacusia de aparición tardía. \\
\hline Preocupación del cuidador respecto a retrasos en la audición, el habla o el desarrollo. ${ }^{*}$ \\
\hline Historia familiar de hipoacusia infantil permanente. ${ }^{*}$ \\
\hline $\begin{array}{l}\text { Estadía en una Unidad de Cuidado Intensivo Neonatal por más de } 5 \text { días, o cualquiera de las siguientes independiente de su duración: } \\
\text { ECMO, ventilación asistida, exposición a medicamentos ototóxicos (gentamicina y tobramicina) o diuréticos de asa (furosemida) e } \\
\text { hiperbilirrubinemia que requiere exanguinotransfusion. }{ }^{*}\end{array}$ \\
\hline Infecciones intrauterinas como: CMV, herpes, rubéola, sífilis, toxoplasmosis. ${ }^{*}$ \\
\hline $\begin{array}{l}\text { Anomalías craneofaciales, incluyendo las del pabellón auricular, conducto auditivo externo, fositas preauriculares y } \\
\text { anomalías del hueso temporal. }\end{array}$ \\
\hline Hallazgos físicos asociados a síndromes que incluyen hipoacusia sensorioneural o de conducción permanentes. \\
\hline $\begin{array}{l}\text { Síndromes asociados a hipoacusia congénita, progresiva o de aparición tardía como neurofibromatosis, osteopetrosis, Usher y otros } \\
\text { síndromes como los de Waardenburg, Alport, Pendred, y Jerverll \& Lange-Nielson. }{ }^{*}\end{array}$ \\
\hline $\begin{array}{l}\text { Desórdenes neurodegenerativos como el síndrome de Hunter o neuropatías sensoriomotrices como la ataxia de Friedrich y el síndrome } \\
\text { de Charcot-Marie-Tooth. }{ }^{*}\end{array}$ \\
\hline $\begin{array}{l}\text { Infecciones posnatales asociadas a HSN con cultivos positivos, incluyendo meningitis bacterianas y virales confirmadas (especialmente } \\
\text { virus herpes y varicela). }{ }^{*}\end{array}$ \\
\hline Traumatismo craneoencefálico, en especial fracturas de base de cráneo y de hueso temporal que requieran hospitalización. ${ }^{*}$ \\
\hline Quimioterapia* \\
Traducido y adaptado de Joint Committee on Infant Hearing. Year 2007 Position Statement: Principles and Guidelines for Early \\
Hearing Detection and Intervention Programs.3 ECMO: Oxigenación con Membrana Extracorpórea, CMV: Citomegalovirus, \\
HSN: Hipoacusia sensorio neural.
\end{tabular}


que regían a la fecha. Es por este motivo que la modificación de la base de datos del PEAN de Clínica Las Condes será una importante intervención, en la medida que podrá organizar la información de forma ordenada y actualizada, y de este modo los casos concurrentes que sean diagnosticados con posterioridad a esta publicación tendrán un registro completo, susceptible de ser evaluado con mayor facilidad y rapidez.

\section{CONCLUSIONES}

Es imperativo la realización de PEAN universales para diagnosticar a la población susceptible a desarrollar $\mathrm{HC}$ ya que se trata de una patología frecuente en nuestra población, con tasas de incidencia que son comparables a las descritas en la literatura y aún más frecuente que aquellas patologías que ya cuentan con screening neonatal universal en nuestro país.

A pesar de que existen múltiples revisiones tanto a nivel local, nacional e internacional, creemos que es fundamental unificar criterios para que los resultados obtenidos sean comparables y extrapolables a diversas poblaciones.

La constante revisión de los resultados del programa ha permitido ir superando las dificultades que se han presentado durante el desarrollo de los protocolos, el advenimiento de nuevas tecnologías y herramientas computacionales permiten que el proceso sea más organizado, facilitando que aquellos recién nacidos referidos reciban precozmente una segunda evaluación, diagnóstico y derivación oportuna.

Comprendemos que la realidad del centro estudiado no corresponde a la realidad nacional, pero entendiendo que el factor socioeconómico no ha sido implicado directamente en el desarrollo de hipoacusia congénita asumimos que la descripción de nuestros resultados será de utilidad para aquellas instituciones que recién inician sus programas de evaluación auditiva neonatal universal.

\section{BIBLIOGRAFÍA}

1. Cornejo V, Raimann e, Cabello Jf, Valiente A, Becerra C, Opazo M, Colombo M. Past, present and future of newborn screnning in Chile. J Inherit
Metab Dis 2010 Aug 4.

2. US Preventive Services Task Force. Universal Screening for Hearing Loss in Newborns: US Preventive Services Task Force Recommendation Statement. Pediatrics Volume 122, Number 1, July 2008.

3. Joint Committee on Infant Hearing. Year 2007 Position Statement: Principles and Guidelines for Early Hearing Detection and Intervention Programs. Pediatrics 2007; 120: 898.

4. Joint Committee on Infant Hearing. Year 2000 Position Statement: Principles and Guidelines for Early Hearing Detection and Intervention Programs. Pediatrics Vol.106 No.4 October 2000.

5. Joint Committee on Infant Hearing. Year 1990 Position Statement. [Revisado el 8 de agosto de 2012]: Disponible en http://www.jcih.org/JCIH1990.pdf.

6. Gonzalo Nazar M, Marcos Goycoolea V, José Miguel Godoy S, Ernesto Ried G, Mónica Sierra G. Evaluación auditiva neonatal universal: Revisión de 10.000 pacientes estudiados. Rev Otorrinolaringol Cir Cabeza Cuello 2009; 69: 93-102.

7. Bielecki I, Horbulewicz A, Wolan T. Risk factors associated with hearing loss in infants: an analysis of 5282 referred neonates. Int $J$ Pediatr Otorhinolaryngol 2011; 75(7): 925-30.

8. Botelho, Marilia Silva e Nunes et al. Newborn hearing screening in the Limiar Clinic in Porto Velho - Rondônia. Braz J Otorhinolaryngol São Paulo, v. 76, n. 5, Oct. 2010.

9. Núñez-Batalla F, Carro-Fernández P, Antuña-León ME, González-Trelles T. Incidencia de hipoacusia secundaria a hiperbilirrubinemia en un programa de cribado auditivo neonatal universal basado en otoemisiones acústicas y potenciales evocados auditivos. Acta Otorrinolaringol Esp 2008; 59(3): 108-13.

10. Martin Ptok. Early Detection of Hearing Impairment in Newborns and Infants. Dtsch Arztebl Int 2011; 108(25): 426-31.

11. Pittaluga E. Guía clínica: Hipoacusia neurosensorial bilateral del prematuro. Gobierno de Chile, Ministerio de Salud. 2009 [Revisado el 8 de agosto de 2012]: Disponible en http://www.supersalud. gob.cl/difusion/572/articles-6447_recurso_1.pdf.

Dirección: Karin Krauss M.

Carmen 390, Depto. 605, Santiago de Chile

Tel: +56 026108667

E mail: karinkrauss@gmail.com 